\title{
Ocorrência de Selenaspidus articulatus (Morgan) (Hemiptera, Diaspididae) e do predador Pentilia egena (Mulsant) (Coleoptera, Coccinelidae) em Myrtus communis L. (Myrtaceae), em Pinheiral, RJ
}

\author{
Edson Henrique de Azeredoํㅜ William Costa Rodrigues ${ }^{2} \&$ Paulo Cesar Rodrigues Cassino $^{3}$
}

\begin{abstract}
'Pró-Reitoria de Extensão, Colégio Agrícola Nilo Peçanha, Universidade Federal Fluminense. Rua José Breves s/n-Centro. 27197-000 Pinheiral-RJ, Brasil. Endereço eletrônico: edsonhenrique.azeredo@bol.com.br

${ }^{2}$ Pós-Graduação em Fitotecnia, Departamento de Fitotecnia, Instituto de Agronomia. Universidade Federal Rural do Rio de Janeiro. 23890-000 Seropédica-RJ, Brasil.

${ }^{3}$ Centro Integrado de Manejo de Pragas Cincinnato Rory Gonçalves, Departamento de Entomologia e Fitopatologia, Instituto de Biologia, Universidade Federal Rural do Rio de Janeiro. 23890-000 Seropédica-RJ, Brasil.
\end{abstract}

\begin{abstract}
Occurrence of Selenaspidus articulatus (Morgan) (Hemiptera, Diaspididae) and of the predator Pentilia egena (Mulsant) (Coleoptera, Coccinelidae) on Myrtus communis L. (Myrtaceae), in Pinheiral County, Rio de Janeiro, Brazil. The evaluations were conducted weekly in the vegetative and blooming/fructification phases in four quadrants $(\mathrm{N}, \mathrm{S}, \mathrm{E}$, W) of the plant. It was collected 2,230 specimens of $S$. articulatus, 1,224 in the vegetative phase and 1,006 in the blooming/fructification, being $59,8 \%$ adults. From the total of 965 of $P$. egena, 416 were immatures and 549 adults. The main conclusions are: (1) M. communis may be indicated as plant bait; (2) this plant, may be cultivated or not around the crops of economic importance, because it may function as a reservoir of the pest or of its natural enemies; (3) the relation predator/prey/plant quadrant/phenology phases was, respectivelly, 1.14:1.0 and 1.0:1,08 in north and east quadrant; while in south and west quadrant was 1.0:2.26 and 1.0:2.80; and, (4) the predaction of $S$. articulatus by P. egena was, in average, 1.0-2.8 nymphs and 1.66-4.44 adults (in vegetative phases) and 1.0-3.76 and 1.0-3.98 (in blooming/ fructification), with significant differences at $\mathrm{P}<0.01$.
\end{abstract}

KEYwords. Biological control; decorative plant; predator-prey relationship; scale insect.

\begin{abstract}
Resumo. As avaliações foram realizadas, semanalmente, durante os estádios vegetativo e de floração/frutificação em quatro quadrantes $(\mathrm{N}, \mathrm{S}, \mathrm{L}, \mathrm{O})$ da planta. Foram coletadas 2.230 espécimes de $S$. articulatus, sendo 1.224 no estágio vegetativo e 1.006 no florescimento/frutificação, sendo 59,8\% adultos. De um total de 965 P. egena, 416 foram imaturos e 549 adultos. As principais conclusões deste trabalho são: (1) M. communis pode ser indicada como planta isca; (2) esta planta pode ser cultivada ou não próxima às culturas de importância econômica, pois funciona como um repositório da praga ou de seu inimigo natural; (3) a relação predador/presa/quadrante da planta/estágio fenológico foi, respectivamente, $1,14: 1,0$ e 1,0:1,08 nos quadrantes norte e leste; enquanto, à sul e oeste da planta $M$. communis foi de 1,0:2,26 e 1,0:2,80; (4) a predação de $S$. articulatus por $P$. egena ocorreu, em média, de 1,0 a 2,8 ninfas e 1,66 a 4,44 adultos (no estágio vegetativo) e de 1,0-3,76 a 1,0-3,98 (no estágio de florescimento/frutificação), com diferenças significativas em $\mathrm{P}<0,01$.
\end{abstract}

Palavras-Chave. Cochonilha; controle biológico; plantas ornamentais; relação predador-presa.

Myrtus communis L., conhecida como "murta”, "bugarin” etc., é um arbusto amplamente utilizado em parques, jardins e vias públicas. Esta Mirtácea é hospedeira de muitos organismos fitófagos, dentre os quais destaca-se a cochonilha Selenaspidus articulatus (Morgan, 1889) (Hemiptera, Diaspididae), considerada praga severa em diversas espécies botânicas.

As cochonilhas podem ocorrer em árvores frutíferas, culturas de ciclo anual, pastagens, essências florestais, ornamentais e em diversas outras espécies vegetais que compõem a biodiversidade de um ecossistema (PrimaVESI 1990). Na cultura da oliveira, Canales-Canales \& Valdivieso-Jara (1999) registraram que $S$. articulatus mostrou alta infestação, sendo controlada por métodos químicos e biológicos. Os danos indiretos causados por $S$. articulatus são devidos às enzimas que injeta antes da sucção da seiva e que possuem alto poder toxicogênico para as plantas (BARTRA 1974; BATISTAFiLHO et al. 1992). Estas enzimas, como a amilase e invertase, causam sintomas típicos de manchas amareladas nas folhas e frutos da planta.

Fatores bióticos e abióticos foram avaliados por CAMPOS (1993) sobre a presença de colônias de S. articulatus em pomares cubanos. Observaram que em folhas de laranjeiras grapefruit, sob temperaturas de 20,25 e $30^{\circ} \mathrm{C}$, atingiram níveis de danos de 19,5; 19,46 e 37,5\%, respectivamente. Altas temperaturas associadas a altas umidades, segundo WATANABE et al. (2000a), propiciam predisposição às plantas cítricas na ocorrência de $S$. articulatus.

De acordo com Watanabe et al. (2000b), realizando amostragens em citros, em diversos municípios paulistas, os níveis econômicos de danos causados por infestação de cochonilhas de carapaças iniciam a partir de 10 indivíduos/ folha. A distribuição espacial e comportamento agregado de S. articulatus, em plantios de laranjeiras foi observada por Perruso \& CAssino (1997), no Brasil e em determinadas regiões 
Tabela I. Período de amostragem, valores totais e freqüências absolutas e relativas (\%) de $S$. articulatus por quadrante durante o estádio vegetativo de M. communis. Pinheiral-RJ, 2000.

\begin{tabular}{|c|c|c|c|c|c|c|c|c|c|c|c|c|c|}
\hline & \multicolumn{8}{|c|}{ Estádio vegetativo } & \multicolumn{4}{|c|}{ Freqüências } & \multirow{3}{*}{$\begin{array}{l}\text { Total } \\
\text { geral }\end{array}$} \\
\hline & \multicolumn{2}{|c|}{$\mathrm{N}^{1}$} & \multicolumn{2}{|c|}{$\mathrm{S}$} & \multicolumn{2}{|r|}{$\mathrm{L}$} & \multicolumn{2}{|c|}{$\mathrm{O}$} & \multicolumn{2}{|c|}{ Absoluta } & \multicolumn{2}{|c|}{ Relativa (\%) } & \\
\hline & $\mathrm{A}$ & B & A & B & A & $\mathrm{B}$ & $\mathrm{A}$ & B & $\mathrm{A}$ & $\mathrm{B}$ & A & B & \\
\hline Março & 27 & 14 & 29 & 38 & 19 & 72 & 23 & 34 & 98 & 158 & 20,63 & 21,10 & 256 \\
\hline Abril & 35 & 88 & 22 & 51 & 26 & 52 & 32 & 56 & 115 & 247 & 24,21 & 32,97 & 362 \\
\hline Maio & 20 & 64 & 31 & 42 & 45 & 41 & 35 & 33 & 131 & 180 & 27,58 & 24,03 & 311 \\
\hline Junho & 32 & 43 & 40 & 15 & 34 & 47 & 25 & 39 & 131 & 164 & 27,58 & 21,90 & 295 \\
\hline Total & 114 & 209 & 122 & 146 & 124 & 212 & 115 & 182 & 475 & 749 & & & 1224 \\
\hline
\end{tabular}

${ }^{1}$ Quadrantes da planta $(\mathrm{N}=$ norte; $\mathrm{S}=$ sul; $\mathrm{L}=$ leste; $\mathrm{O}=$ oeste). $\mathrm{A}=$ ninfa; $\mathrm{B}=$ adulto

de Cuba (SuRIS 1999).

Em outubro de 1996, na cidade de Bebedouro (SP), algumas plantas de Mimosa caesalpiniaefolia (Benth.) (Leguminosae) usadas como uma cerca viva, foram infestadas por $S$. articulatus. Estas observações corroboram que cercas vivas podem influenciar a incidência de insetos-praga em pomares de citros (LARANJEIRA 1996).

Fontenla Rizo (1990) estudando seis espécies da superfamília Coccoidea afetando pomares de citros em Cuba, entre 1986/87, constatou abundância máxima entre outubro e novembro para Diaspididae, e fevereiro para Coccidae, além da existência de guilda ecológica e vivendo em competição interespecífica, assim como sugerem que o controle do inseto em comunidade foi mais eficiente. Em cafeeiros, na região de Chiapar, no México, foram tomadas amostragens com três e 14 dias de intervalos entre novembro/1983 e abril/1985 (IBARRANUNEs 1990). Segundo estes autores, em 37 amostragens foram registradas 609 espécies de artrópodes (em 258 famílias), dentre elas, S. articulatus, em abundância e considerada praga potencial.

Selenaspidus articulatus foi observada danificando plantas de seringueira em São Paulo, pela primeira vez, por Bergmann et al. (1988). Pomares contendo laranja, lima, grapefruit e limão, em Cuba, foram pesquisados (HERNANDEZ \& CeBALLos 1987) e se mostraram atacados por S. articulatus, parasitados por diversos hemípteros. A distribuição espacial de $S$. articulatus em plantação de laranjas foi estudada durante 1981/83 (Suris \& VARONa 1988), em Cuba, mostrando a distribuição da praga ser heterogênea.

WatANABE et al. (1994) afirmam que um dos fatores que contribuem para a proliferação de $S$. articulatus está na destruição dos inimigos naturais, provocado pelo uso abusivo de agrotóxicos.

A eficiência do Manejo Integrado de Pragas como estratégia do controle de pragas foi comprovada por GALLI et al. (1995) em relação à Phyllocoptruta oleivora (Ashmead, 1979), S. articulatus e Toxoptera citricidus (Kirk., 1907) em pomares de laranjeiras em São Paulo, no período de dezembro de 1992 a abril de 1993.

Dentre os predadores de $S$. articulatus, os coccinelídeos são considerados os mais importantes. Dos seus representantes, Pentilia egena (Mulsant, 1850) é considerado predador ágil exercendo importante controle biológico sobre algumas pragas e o principal inimigo natural daquela espécie em plantas cítricas (Watanabe 1996,1999). Segundo WatanABe (1999), a longevidade dos machos adultos é, em média, 111 dias e das fêmeas, 103 dias. Estudos realizados em laboratório mostraram que os machos de $S$. articulatus atingem maturidade sexual aos 3,2 \pm 2 dias (Hoz-Gonzalez 1995). $P$. egena, na fase adulta, alimenta-se, por dia, de até $17 \mathrm{~S}$. articulatus adultos ou mais de 90 ninfas (Gravena 1999).

Do ponto de vista ambiental, torna-se importante o incremento nos estudos relacionados ao comportamento de $P$. egena no controle de $S$. articulatus e outras cochonilhas em plantas ornamentais. Segundo WiLlinK \& Claps (2003), nos últimos anos aumentou consideravelmente a produção e

Tabela II. Período de amostragem, valores totais e freqüências absolutas e relativas (\%) de S. articulatus por quadrante durante o estádio de florescimento/frutificação de M. communis. Pinheiral-RJ, 2000.

\begin{tabular}{|c|c|c|c|c|c|c|c|c|c|c|c|c|c|}
\hline \multirow{3}{*}{$\begin{array}{l}\text { Período de } \\
\text { amostragem }\end{array}$} & \multicolumn{8}{|c|}{ Estádio de florescimento/ frutificação } & \multicolumn{4}{|c|}{ Freqüências } & \multirow{3}{*}{$\begin{array}{l}\text { Total } \\
\text { geral }\end{array}$} \\
\hline & \multicolumn{2}{|c|}{$\mathrm{N}^{1}$} & \multicolumn{2}{|c|}{$\mathrm{S}$} & \multicolumn{2}{|c|}{$\mathrm{L}$} & \multicolumn{2}{|c|}{$\mathrm{O}$} & \multicolumn{2}{|c|}{ Absoluta } & \multicolumn{2}{|c|}{ Relativa (\%) } & \\
\hline & $\mathrm{A}$ & $\mathrm{B}$ & A & $\mathrm{B}$ & $\mathrm{A}$ & $\mathrm{B}$ & $\mathrm{A}$ & $\mathrm{B}$ & $\mathrm{A}$ & $\mathrm{B}$ & $\mathrm{A}$ & $\mathrm{B}$ & \\
\hline Julho & 11 & 44 & 28 & 39 & 11 & 14 & 4 & 27 & 54 & 124 & 12,80 & 21,23 & 178 \\
\hline Agosto & 13 & 57 & 52 & 50 & 21 & 25 & 22 & 50 & 108 & 182 & 25,59 & 31,16 & 290 \\
\hline Setembro & 24 & 67 & 20 & 26 & 24 & 41 & 36 & 18 & 104 & 142 & 24,64 & 24,31 & 246 \\
\hline Outubro & 10 & 55 & 61 & 34 & 34 & 24 & 51 & 23 & 156 & 136 & 36,97 & 23,30 & 292 \\
\hline Total & 58 & 223 & 161 & 149 & 90 & 94 & 113 & 118 & 422 & 584 & & & 1006 \\
\hline
\end{tabular}

${ }^{1}$ Quadrantes da planta $(\mathrm{N}=$ norte; $\mathrm{S}=\mathrm{sul} ; \mathrm{L}=$ leste; $\mathrm{O}=$ oeste). $\mathrm{A}=$ ninfa; $\mathrm{B}=$ adulto. 
Tabela III. Período de amostragem e dados da contingência ( 2 x 4 ) dos valores observados e esperados e total geral no estágio ninfal e adulto de $S$. articulatus, durante o estádio vegetativo em $M$. communis. Pinheiral-RJ, 2000.

\begin{tabular}{lcccccc}
\hline \multirow{2}{*}{$\begin{array}{l}\text { Período } \\
\text { de amos- }\end{array}$} & \multicolumn{4}{c}{ S. articulatus } & \multirow{2}{*}{$\begin{array}{c}\text { Total } \\
\text { geral }\end{array}$} \\
\cline { 2 - 5 } \cline { 5 - 6 } \cline { 5 - 6 } & \multicolumn{2}{c}{ Ninfa } & & \multicolumn{2}{c}{ Adulto } & \\
\cline { 2 - 5 } Março & 98 & 4 & & 158 & 99,35 & 256 \\
Abril & 115 & 110 & & 247 & 205,35 & 362 \\
Maio & 131 & 59 & & 180 & 154,35 & 311 \\
Junho & 131 & 43 & & 164 & 138,35 & 295 \\
\hline Total & 475 & 216 & 749 & 417,40 & 1224 \\
\hline
\end{tabular}

$\chi_{\mathrm{o}}^{2}=153,69>\chi_{\mathrm{c}}^{2}=11,34 ; \mathrm{P}=0,01 ; \mathrm{Gl}=3$.

comercialização de plantas exóticas e autóctones, assim como a importação e exportação de numerosas plantas e flores que muitas vezes não possuem controle fitossanitário. Isto favorece a introdução de novas pragas e a proliferação de outras devido às condições ambientais em que se desenvolvem, diferindo de outras que, em condições naturais, não seriam prejudiciais. Entre as 53 plantas hospedeiras visitadas por $S$. articulatus (WILLIAMS \& WATSON 1988), não foram encontradas informações sobre M. communis, evidenciando a necessidade de diagnosticar parâmetros da interação predador-presa.

Desse modo, o objetivo do presente trabalho foi avaliar a ocorrência de $S$. articulatus e do predador $P$. egena em $M$. communis, através de amostragens realizadas nos diversos quadrantes da planta. Além disso, devido à escassez de estudos sobre a relação de predação entre $P$. egena e $S$. articulatus, diagnosticar as freqüências relativas dessas espécies nos diversos estádios de desenvolvimento de $M$. communis.

\section{MATERIAL E MÉTODOS}

O trabalho foi realizado no Colégio Agrícola Nilo Peçanha da Universidade Federal Fluminense em Pinheiral, Rio de

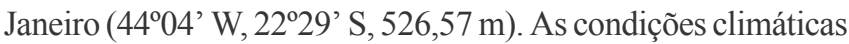
foram temperatura máxima $28,8 \pm 0,78^{\circ} \mathrm{C}$, temperatura mínima $10,51 \pm 1,14^{\circ} \mathrm{C}$ e umidade relativa do ar $82,1 \pm 1,96 \%$. Foram utilizadas três matrizes de $M$. communis onde foram efetuadas as leituras de distribuição de $S$. articulatus e a ocorrência de P. egena. Visando a obtenção de informações sobre a relação de predação de $P$. egena sobre $S$. articulatus, foram considerados os estádios de desenvolvimento vegetativo e de florescimento/frutificação como parâmetros, no período de março a outubro de 2000. Como critério, as alturas das amostragens foram estabelecidas em 0,8; 1,30 e 1,80 m com as seqüências de leituras no sentido colo-ápice de cada ramo. Em cada amostragem foram coletados três folíolos nos diferentes quadrantes da planta (norte, sul, leste e oeste), num total de 108 folíolos amostrados.

Para determinação do número de ninfas e adultos de $S$. articulatus por folíolo, foi utilizada uma lente de bolso Modelo PMO-Oranienburg DDR-10 dpt e Kend Rufast, adaptada em um vazador da folha, específico para o estudo dessa espécie, com área de $2 \mathrm{~cm}^{2}$, determinando-se as freqüências absolutas e relativas. As observações foram feitas entre 10 e 14 horas.

O mesmo procedimento foi adotado para avaliar o número de larvas/pupas e adultos de $P$. egena. Após calculadas as freqüências absolutas e relativas, foi elaborada uma matriz de predação para estudos da relação predador-presa. Os levantamentos (presença-ausência) de $S$. articulatus (ninfas e adultos) e $P$. egena foram realizados examinando-se as duas superfícies dos folíolos de M. communis.

Posteriormente, foram elaboradas tabelas de contingência para averiguar diferenças existentes entre adultos, ninfas e imaturos e os respectivos totais observados e esperados para as espécies estudadas, utilizando-se a correção de Yates segundo Levin ( 1978 ). Para análise estatística, os dados foram submetidos ao teste de significância do Qui-quadrado $\left(\chi^{2}\right)$ (LEVIN 1978 ), ao nível de 5\% de probabilidade.

\section{RESULTADOS EDISCUSSÃO}

Foram coletados 1.224 indivíduos de $S$. articulatus no estádio vegetativo de $M$. communis, sendo 475 ninfas e 749 adultos, nos quatro quadrantes da planta (Tabela I). No mês de abril foi registrado o maior pico populacional da cochonilha (362 espécimes) durante o estádio vegetativo da planta, com uma proporção de 2,1 adultos para cada ninfa, reduzindo-se no período de maio a junho, com 1,4 e 1,3 adultos para cada ninfa, respectivamente (Tabela I e Fig.1).

O menor pico populacional de $S$. articulatus ocorreu no estádio de florescimento/ frutificação, no mês de julho (com 178 espécimes) (Fig.2) representando 17,7\% do total da amostragem (Tabela II). A flutuação populacional de $S$. articulatus e a influência da temperatura e precipitação foram estudadas por PERRUSO \&CASSINO (1993) que observaram uma menor infestação entre os meses de março a julho, quando a temperatura e precipitação apresentaram índices reduzidos. A população da praga teve incremento no final do inverno (agosto) com o aumento das temperaturas, e novamente entre

Tabela IV. Período de amostragem e dados da contingência ( 2 x 4 ) dos valores observados e esperados e total geral no estágio ninfal e adulto de $S$. articulatus, durante o estádio de florescimento/frutificação em $M$. communis. Pinheiral-RJ, 2000.

\begin{tabular}{|c|c|c|c|c|c|}
\hline \multirow{3}{*}{$\begin{array}{l}\text { Período } \\
\text { de amos- } \\
\text { tragem }\end{array}$} & \multicolumn{4}{|c|}{ S. articulatus } & \multirow{3}{*}{$\begin{array}{l}\text { Total } \\
\text { geral }\end{array}$} \\
\hline & \multicolumn{2}{|c|}{ Ninfa } & \multicolumn{2}{|c|}{ Adulto } & \\
\hline & Observado & Esperado & Observado & Esperado & \\
\hline Julho & 54 & 49,28 & 124 & 74,67 & 178 \\
\hline Agosto & 108 & 161,28 & 182 & 186,67 & 290 \\
\hline Setembro & 104 & 117,28 & 142 & 142,67 & 246 \\
\hline Outubro & 156 & 163,28 & 136 & 188,67 & 292 \\
\hline Total & 422 & 491,12 & 584 & 592,68 & 1006 \\
\hline
\end{tabular}

$\chi_{\mathrm{o}}^{2}=66,26>\chi_{\mathrm{c}}^{2}=11,34 ; \mathrm{P}=0,01 ; \mathrm{Gl}=3$ 


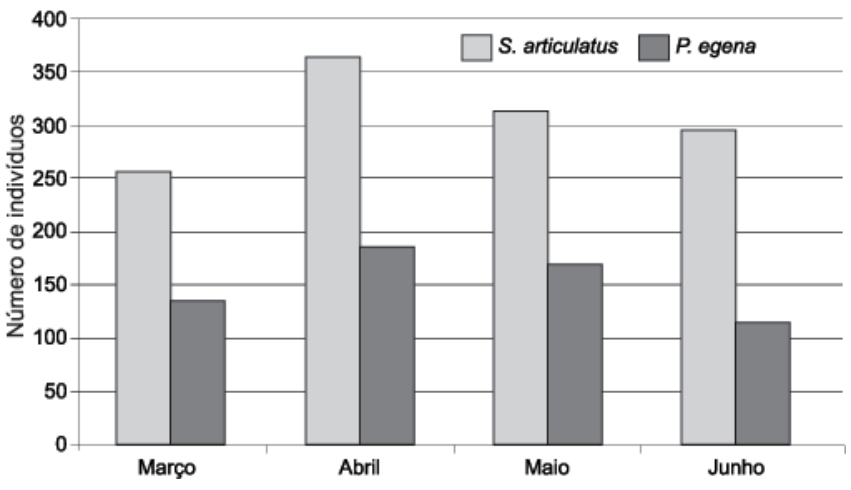

Fig. 1. Comportamento populacional de S. articulatus e P. egena em plantas de M. communis em estádio vegetativo, entre os meses de março e junho em Pinheiral-RJ, 2000.

setembro e janeiro com a elevação da temperatura e precipitação.

Em relação à distribuição espacial observa-se, na Tabela II, um aumento no número de cochonilhas nos ramos dos quadrantes norte e sul da planta hospedeira isto é, um total de 219 e 372 indivíduos (ninfas e adultos), respectivamente. Os maiores picos populacionais foram registrados nos meses de agosto e outubro, com 290 e 292 espécimes. O mesmo fato foi observado nos quadrantes leste e oeste, porém, em menor proporção (203 e 212), diferindo do observado por LOAYZA \& PARRA (2001) que, em relação à posição de maior incidência de S. articulatus em Citrus $\mathbf{s p}$., verificaram que a face de exposição norte e a parte interna da planta ofereceram melhores condições para a sobrevivência das cochonilhas. Além disso, constataram que a concentração da praga foi mais intensa na face superior da folha, próxima ao pecíolo.

LOAYZA \& PARRA (2001), em plantas cítricas, constataram que a preferência de $S$. articulatus, em geral, é por folhas mais velhas onde foi verificada a maior infestação de indivíduos de S. articulatus $(53,8 \%)$. No presente trabalho, durante o estádio de florescimento/frutificação foi observada uma redução na população de $S$. articulatus ( Tabela II). O número de ninfas e adultos de $S$. articulatus, mostrou diferenças significativas

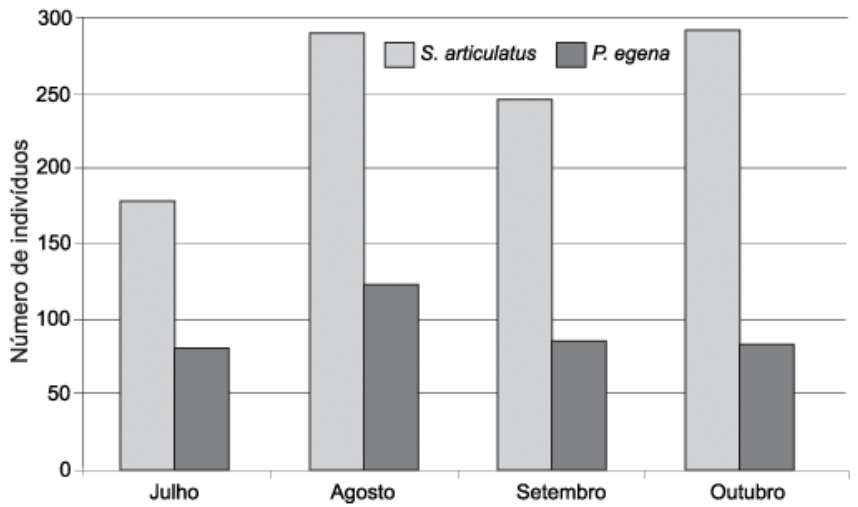

Fig. 2. Comportamento populacional de S. articulatus e P. egena em plantas de M. communis em estádio de florescimento / frutificação, entre os meses de julho e outubro em Pinheiral-RJ, 2000.

através do Qui-quadrado observado $\left(\chi^{2}\right)$ de 153,$69 ; \mathrm{P}=0,01$ e g.l. $=3$, quando o total de freqüências esperadas tanto de ninfa quanto de adultos foram, aproximadamente, $50 \%$ de seus totais, demonstrando que a praga está bem adaptada nutricionalmente à planta (Tabela III).

A dinâmica populacional de $S$. articulatus, durante o estádio vegetativo de $M$. communis apresentou uma amostragem seqüencial de 1.224 espécimes, sendo 475 ninfas e 749 adultos (Tabela III). Observou-se, através dos dados da contingência, que o número de ninfas e de adultos amostrados superou as freqüências esperadas. O número de ninfas de $S$. articulatus variou de 98 a 131 exemplares, enquanto o de cochonilhas adultas variou de 158 a 247 , principalmente nas amostragens realizadas no período de março a abril de 2000. No entanto, os níveis populacionais de ninfas mostrara-se reduzidos em março, porém ocorreu pico populacional de adultos em abril, com um total de 247 espécimes, ou 29,58\% do total de cochonilhas amostradas (1.224 espécimes) (Tabela III).

A partir do mês de julho de 2000, os picos populacionais de $S$. articulatus no estádio de florescimento/frutificação de M. communis, demonstraram tendência à estabilização ou à redução no número de adultos, registrando 182, 142 e 136

Tabela V. Período de amostragem, número médio de P. egena na predação de $S$. articulatus, no estádio vegetativo e quadrante de $M$. communis. Pinheiral-RJ, 2000.

\begin{tabular}{|c|c|c|c|c|c|c|c|c|c|c|c|c|c|}
\hline \multirow{3}{*}{$\begin{array}{l}\text { Período } \\
\text { de amost. }\end{array}$} & \multicolumn{12}{|c|}{ P. egena } & \multirow{3}{*}{$\begin{array}{l}\text { Total } \\
\text { geral }\end{array}$} \\
\hline & \multicolumn{6}{|c|}{ Larva/pupa } & \multicolumn{6}{|c|}{ Adulto } & \\
\hline & $\mathrm{N}^{1}$ & $\mathrm{~S}$ & $\mathrm{~L}$ & $\mathrm{O}$ & Freq. abs. & Freq. rel. (\%) & $\mathrm{N}$ & $\mathrm{S}$ & $\mathrm{L}$ & $\mathrm{O}$ & Freq. abs. & Freq. rel. (\%) & \\
\hline Março & 12 & 7 & 11 & 9 & 39 & 14,66 & 41 & 18 & 22 & 14 & 95 & 28,27 & 134 \\
\hline Abril & 31 & 27 & 19 & 16 & 93 & 34,96 & 37 & 12 & 40 & 3 & 92 & 27,38 & 185 \\
\hline Maio & 26 & 18 & 18 & 24 & 86 & 32,33 & 32 & 14 & 26 & 11 & 83 & 24,70 & 169 \\
\hline Junho & 17 & 10 & 14 & 7 & 48 & 18,04 & 16 & 10 & 27 & 13 & 66 & 19,64 & 114 \\
\hline Total & 86 & 62 & 62 & 56 & 266 & 100 & 126 & 54 & 115 & 41 & 336 & 100 & 602 \\
\hline
\end{tabular}

${ }^{1}$ Quadrantes da planta $(\mathrm{N}=$ norte; $\mathrm{S}=$ sul; $\mathrm{L}=$ leste; $\mathrm{O}=$ oeste $)$. 
Tabela VI. Período de amostragem, número médio de $P$. egena na predação de $S$. articulatus, no estádio de florescimento/frutificação e quadrante de M. communis. Pinheiral-RJ, 2000.

\begin{tabular}{|c|c|c|c|c|c|c|c|c|c|c|c|c|c|}
\hline \multirow{3}{*}{$\begin{array}{l}\text { Período } \\
\text { de amost. }\end{array}$} & \multicolumn{12}{|c|}{ P. egena } & \multirow{3}{*}{$\begin{array}{l}\text { Total } \\
\text { geral }\end{array}$} \\
\hline & \multicolumn{6}{|c|}{ Larva/pupa } & \multicolumn{6}{|c|}{ Adulto } & \\
\hline & $\mathrm{N}^{1}$ & $\mathrm{~S}$ & $\mathrm{~L}$ & $\mathrm{O}$ & Freq. abs. & Freq. rel. (\%) & $\mathrm{N}$ & $\mathrm{S}$ & $\mathrm{L}$ & $\mathrm{O}$ & Freq. abs. & Freq. rel. (\%) & \\
\hline Julho & 7 & 6 & 9 & 4 & 26 & 16,25 & 21 & 13 & 12 & 9 & 55 & 25,82 & 81 \\
\hline Agosto & 12 & 14 & 23 & 16 & 65 & 40,62 & 16 & 9 & 24 & 10 & 59 & 27,70 & 124 \\
\hline Setembro & 14 & 13 & 10 & 1 & 38 & 23,75 & 18 & 13 & 12 & 4 & 47 & 22,06 & 85 \\
\hline Outubro & 7 & 11 & 9 & 4 & 31 & 19,37 & 15 & 10 & 20 & 7 & 52 & 24,41 & 83 \\
\hline Total & 40 & 44 & 51 & 25 & 160 & 100 & 70 & 45 & 68 & 30 & 213 & 100 & 373 \\
\hline
\end{tabular}

${ }^{1}$ Quadrantes da planta $(\mathrm{N}=$ norte; $\mathrm{S}=$ sul; $\mathrm{L}=$ leste; $\mathrm{O}=$ oeste $)$.

cochonilhas (Tabela IV). Este comportamento parece ocorrer em função das transformações fisiológicas da planta decorrentes da sazonalidade de compostos nutricionais. O efeito desse comportamento é refletido pela predisposição à população de $S$. articulatus que demonstrou agregação definida de ninfas de $3^{\circ}$ e $4^{\circ}$ ínstares, diferindo do adulto que mostrou declínio no número de exemplares. Isto foi provocado, possivelmente, pelo estádio de florescimento/frutificação que tornou o ambiente menos atrativo à cochonilha $\left(\chi^{2}=66,26>\right.$ $\chi_{\mathrm{c}}^{2}=11,34, \mathrm{P}=0,01 \mathrm{e} \mathrm{gl}=3$ ) (Tabela IV).

Constatou-se ainda que, no estágio ninfal, principalmente durante o mês de julho, as ninfas de $S$. articulatus apresentaram um pico populacional $50 \%$ menor, confirmando a tendência dos dados registrados na Tabela III. Já, na Tabela $\mathrm{V}$, foram registrados 602 exemplares de P. egena, 266 imaturos e 336 adultos durante o período (Tabela V).

Em relação aos quadrantes da planta foram observados, ao norte, 212 indivíduos, 86 imaturos e 126 adultos de P. egena. No entanto, as amostragens foram diferenciadas em cada período de leitura. Para larvas/pupas os picos populacionais ocorreram no período de abril a maio, com 93 e 86 exemplares, respectivamente, diferindo dos adultos com valores, em março e abril, de 95 e 92 indivíduos (Tabela V). Este fato pode ser explicado pela necessidade do predador conviver no ambiente de M. communis com um limite mínimo de presas, o que é corroborado com a Tabela VII, onde os valores esperados das freqüências de ninfas e de adultos mostraram-se reduzidos em $50 \%$. Verifica-se, ainda, que durante o mês de maio de 2000 ocorreu um ligeiro aumento no valor observado de larvas/ pupas de $P$. egena (Tabela V).

As condições climáticas e ambientais de Pinheiral permitiram que, durante o estádio de desenvolvimento vegetativo da planta, houvesse freqüência estável do inseto durante todo o período de amostragem, excetuando-se apenas os indivíduos coletados em junho, quando foram registrados 66 espécimes. Tendência idêntica foi observada com as larvas/ pupas com redução em 56\% dos 602 exemplares encontrados durante o estádio vegetativo. Não se observou, porém, essa tendência, durante a fase de florescimento/frutificação (Tabela VI), quando houve redução de $P$. egena (larvas/pupas), nos dois últimos meses de amostragem (setembro-outubro) apresentando, respectivamente, 38 e 31 exemplares, e com valor do Qui-quadrado observado $\left(\chi_{0}^{2}=54,44 \%\right)$, superior ao valor do Qui-quadrado crítico.

Com relação à posição de ataque da praga na planta, $o$ quadrante leste, nos dois estágios de desenvolvimento de $P$. egena, atingiu os maiores valores (51 e 68) para larvas/pupas e adultos, respectivamente.
Tabela VII. Período de amostragem e dados da contingência $(2 \times 4)$ dos valores observados e esperados, e total geral de $P$. egena, durante o estádio vegetativo em M. communis. Pinheiral-RJ, 2000.

\begin{tabular}{lcccccr}
\hline \multirow{2}{*}{$\begin{array}{l}\text { Período } \\
\text { de amos- } \\
\text { tragem }\end{array}$} & \multicolumn{4}{c}{ Larva/pupa } & & \multicolumn{2}{c}{ Adulto } & \multirow{2}{*}{$\begin{array}{c}\text { Total } \\
\text { geral }\end{array}$} \\
\cline { 2 - 3 } \cline { 5 - 6 } & $\begin{array}{c}\text { Valor } \\
\text { observado }\end{array}$ & $\begin{array}{c}\text { Valor } \\
\text { esperado }\end{array}$ & & $\begin{array}{c}\text { Valor } \\
\text { observado }\end{array}$ & $\begin{array}{c}\text { Valor } \\
\text { esperado }\end{array}$ & \\
\hline Março & 39 & 45,74 & 95 & 59,61 & 134 \\
Abril & 93 & 96,40 & 92 & 110,21 & 185 \\
Maio & 86 & 80,74 & & 83 & 94,21 & 169 \\
Junho & 48 & 25,40 & & 66 & 39,21 & 114 \\
\hline Total & 266 & 248,28 & 336 & 303,24 & 602 \\
\hline
\end{tabular}

$\chi_{\mathrm{o}}^{2}=50,48>\chi_{\mathrm{c}}^{2}=11,34 ; \mathrm{P}=0,01 ; \mathrm{GL}=3$.
Tabela VIII. Período de amostragem e dados da contingência $(2 \times 4)$ dos valores observados e esperados, e total geral de $P$. egena, durante o estádio de florescimento/frutificação em M. communis. Pinheiral-RJ, 2000.

\begin{tabular}{lcccccc}
\hline \multirow{2}{*}{$\begin{array}{l}\text { Período } \\
\text { de amos- }\end{array}$} & \multicolumn{4}{c}{ P. egena } \\
\cline { 2 - 3 } tragem & \multicolumn{2}{c}{ Larva/pupa } & & \multicolumn{2}{c}{ Adulto } & \\
\cline { 2 - 3 } & $\begin{array}{c}\text { Valor } \\
\text { observado }\end{array}$ & $\begin{array}{c}\text { Valor } \\
\text { esperado }\end{array}$ & & $\begin{array}{c}\text { Valor } \\
\text { observado }\end{array}$ & $\begin{array}{c}\text { Valor } \\
\text { esperado }\end{array}$ & \\
\hline Julho & 26 & 20,39 & & 55 & 34,75 & 81 \\
Agosto & 65 & 63,39 & & 59 & 77,75 & 124 \\
Setembro & 38 & 24,39 & & 47 & 38,75 & 85 \\
Outubro & 31 & 22,39 & 52 & 36,75 & 83 \\
\hline Total & 160 & 130,56 & 213 & 188,00 & 373 \\
\hline
\end{tabular}

$\chi_{\mathrm{o}}^{2}=54,44>\chi_{\mathrm{c}}^{2}=11,34 ; \mathrm{P}=0,01 ; \mathrm{GL}=3$ 
Tabela IX. Quadrante e relação de predação de adultos de $P$. egena sobre ninfas e adultos de $S$. articulatus, no estágio vegetativo de $M$. communis. Pinheiral-RJ, 2000.

\begin{tabular}{|c|c|c|c|c|c|}
\hline \multirow{2}{*}{$\begin{array}{l}\text { Quadrante de } \\
\text { M. communis } \\
\text { monitorado }\end{array}$} & \multirow{2}{*}{$\frac{\text { P. egena }}{\text { Adulto }}$} & \multicolumn{4}{|c|}{ S. articulatus } \\
\hline & & Ninfa & Rp. ${ }^{1}$ & Adulto & Rp. \\
\hline $\mathrm{N}^{2}$ & 126 & 114 & $1,14: 1,0$ & 209 & $1,0: 1,66$ \\
\hline $\mathrm{S}$ & 54 & 122 & $1,0: 2,26$ & 146 & $1,0: 2,70$ \\
\hline $\mathrm{L}$ & 115 & 124 & $1,0: 1,08$ & 212 & $1,0: 1,84$ \\
\hline $\mathrm{O}$ & 41 & 115 & $1,0: 2,80$ & 182 & $1,0: 4,44$ \\
\hline Total & 336 & 475 & & 749 & \\
\hline
\end{tabular}

${ }^{1} \mathrm{Rp} .=$ Relação predador: presa.

${ }^{2}$ Quadrantes da planta $(\mathrm{N}=$ norte; $\mathrm{S}=$ sul; $\mathrm{L}=$ leste; $\mathrm{O}=$ oeste $)$.

O comportamento dos adultos de $P$. egena mostrou uma redução do pico populacional em 25,4\%. Comparando-se os dados às Tabelas VII e VIII observa-se que esta espécie demonstrou adaptar-se na região Sul-Fluminense, pois os espécimes amostrados registraram valores de $\chi^{2}{ }_{0}$ sempre acima dos esperados no campo, variando de 86-48 (nos meses de maio e junho) e 95-66 ( em março e junho) (Tabela VII) (Fig.1). No entanto, durante o período de florescimento/frutificação (Tabela VIII) (Fig.2), entre os meses de julho e outubro, todos os valores observados, principalmente de larvas/pupas, foram acima dos esperados, enquanto no estágio adulto a freqüência foi menor no mês de agosto, indicando ser o período de menor ocorrência da presa (S. articulatus) e, possivelmente, de transformações na estrutura vegetal da planta devido às condições climáticas, o que propiciou o desenvolvimento mais lento do predador (P. egena).

A conservação e manutenção da população de inimigos naturais no campo é, segundo Rodrigues e CASSINo (2004), uma alternativa do controle biológico, onde aproveitam-se os inimigos naturais existentes no agroecossistema e através de manejo cultural, tenta-se manter e até aumentar a população destes inimigos naturais no pomar. Outra forma é utilizar o programa de Manejo Integrado de Pragas onde as aplicações de agroquímicos respeitam todo um critério de equilíbrio populacional o que é conseguido com monitoramentos periódicos.

Quando se avaliou estatisticamente a ocorrência de $P$. egena no estágio vegetativo da planta, foi encontrado um valor do Qui-quadrado de 50,48 a 1\% de probabilidade ( Tabela VII). Na Tabela VIII encontram-se os valores das freqüências observadas e esperadas de $P$. egena (larvas/pupa e adulto), cujo valor $\chi_{0}^{2}$ é de 54,44 para o estágio de florescimento/ frutificação.

Os resultados da contingência mostraram que as freqüências esperadas diferiram das observadas (Tabela VII) pois, de acordo com os dados, esperavam-se no mês de março, aproximadamente, 59,61 adultos de $P$. egena, sendo registrados 95 indivíduos, fato também observado em junho, quando 39,21 indivíduos eram esperados, para 66 registrados. Em relação às larvas/pupas, no mês de junho, o resultado esperado foi de 25,4 exemplares sendo, no entanto, observados 48 indivíduos.
Esses resultados podem estar relacionados a muitos fatores que, possivelmente, propiciaram a concentração da cochonilha nesse estágio fenológico da planta (Tabela VIII), o que mostra que a maior disponibilidade de presas é que deve ter favorecido o aumento populacional do predador.

De forma geral, avaliando-se a capacidade de predação de P. egena (adulto) sobre $S$. articulatus (ninfa e adulto) foi observado que, durante o estágio vegetativo e em relação ao quadrante avaliado de $M$. communis, ocorreu um comportamento diferenciado do predador (Tabela IX). A relação de predação foi menos intensa nos quadrantes norte $\mathrm{e}$ leste com, respectivamente, 1,14:1 e 1:1,08. Estes dados indicam que uma das variáveis de influência na relação predador/presa e comportamento das espécies foi a posição da planta hospedeira. De acordo com Oметто (1981), o fotoperiodo com insolação máxima possível é um parâmetro não só importante dentro do ponto de vista fisiológico, sendo atuante em processos fotossintéticos e morfológicos em uma planta, mas estabelecendo diferentes atividades em insetos e aracnídeos predadores. Em artrópodes e insetos predadores, o fotoperiodismo ocasiona infestações diferenciais no decorrer do ano, estabelecendo os de hábitos diurnos e noturnos.

Avaliando a taxa de predação em folhas de algodoeiro, OLIVEIRA et al. (2001) verificaram que a espécie hospedeira e fatores climáticos, são o diferencial do comportamento do biocontrolador. Também observaram que o predador necessita explorar o máximo de área foliar para encontrar sua presa e que a taxa de predação aumenta com o aumento da densidade da presa.

De acordo com Letourneau \& Altieri (1983), citados por Mendes et al. (2003), população de predadores mais complexos devido à maior disponibilidade de recursos alimentares e, pelo fato de não ser dependente muitas vezes de uma espécie de presa, pode permanecer no ambiente prevenindo a infestação da presa-alvo.

GLiessman (2001) observou que os organismos estão em competição pelos recursos do espaço e não pelo espaço em si. Assim, os parasitas dependem fisiológicamente de seus hospedeiros, têm vida mais curta e alto potencial reprodutor. Em termos nutricionais, a exposição de plantas de cafeeiro

Tabela X. Quadrante e relação de predação de adultos de $P$. egena sobre ninfas e adultos de $S$. articulatus, no estágio de florescimento/ frutificação de M. communis. Pinheiral-RJ, 2000.

\begin{tabular}{|c|c|c|c|c|c|}
\hline \multirow{2}{*}{$\begin{array}{l}\text { Quadrante de } \\
M \text {. communis } \\
\text { monitorado }\end{array}$} & \multirow{2}{*}{$\begin{array}{r}\text { P. egena } \\
\text { Adulto }\end{array}$} & \multicolumn{4}{|c|}{ S. articulatus } \\
\hline & & Ninfa & Rp. ${ }^{1}$ & Adulto & Rp. \\
\hline$\overline{\mathrm{N}^{2}}$ & 70 & 58 & $1,20: 1,0$ & 223 & $1,0: 3,18$ \\
\hline $\mathrm{S}$ & 45 & 161 & $1,0: 3,57$ & 149 & $1,0: 3,31$ \\
\hline $\mathrm{L}$ & 68 & 90 & $1,0: 1,32$ & 94 & $1,0: 1,38$ \\
\hline $\mathrm{O}$ & 30 & 113 & $1,0: 3,76$ & 118 & $1,0: 3,98$ \\
\hline Total & 213 & 422 & & 584 & \\
\hline
\end{tabular}

${ }^{1} \mathrm{Rp} .=$ Relação predador: presa.

${ }^{2}$ Quadrantes da planta $(\mathrm{N}=$ norte; $\mathrm{S}=\mathrm{sul} ; \mathrm{L}=$ leste; $\mathrm{O}=$ oeste $)$. 
parece não influenciar o teor dos elementos nas folhas (Malavolta et al. 1997), embora as folhas voltadas para o oeste, em geral, mostram mais intensamente sintomas de carência (de boro e zinco, principalmente). Para diminuir o erro na variação, esses autores recomendam colher as amostras nas quatro exposições (N, S, L e O) em plantas à meia-sombra. Nos quadrantes sul e oeste de $M$. communis, o efeito da predação foi mais visível (Tabela IX), verificando-se que a capacidade de predação de $P$. egena sobre adultos de $S$. articulatus foi superior, ou seja, apresentando 1,0:2,26 e $1,0: 2,80$, respectivamente.

A relação de predação de $P$. egena sobre $S$. articulatus (Tabelas IX e X) variou, em média, de 1,0 a 2,8 ninfas e 1,66 a 4,44 adultos (no estágio vegetativo) e de 1,0 a 3,76 a 1,0 e 3,98 (no estágio de florescimento/frutificação), respectivamente nos diversos quadrantes de $M$. communis, mostrando em condições naturais que o predador apresentava influência sobre a presa assim que a planta hospedeira atingiu a idade fisiológica.

Com base nos resultados da Tabela X, confirmam-se as tendências de que os quadrantes norte e leste de $M$. communis, no estágio fenológico de florescimento/frutificação não propiciaram capacidade de predação, isto é, 1,20 predadores para 1,0 presa (em relação à população de ninfas), cujos folíolos avaliados apresentaram maior população de $P$. egena. Já na predação de adultos de $S$. articulatus, a relação neste mesmo quadrante foi de 3,18 presas por predador. Houve, entretanto, no quadrante leste, a primeira relação de predação de $P$. egena, tanto sobre ninfas quanto de adultos de $S$. articulatus. Entretanto, os quadrantes sul e oeste de M. communis foram os substratos com maior eficácia de predação.

Provavelmente, o processo de predação da cochonilha tenha ocorrido de forma diferencial devido à planta encontrarse em idade fisiológica de senescência alterando a fisiologia da planta em função de distúrbios metabólicos que naturalmente ocorrem, tornando-a menos atrativa à $S$. articulatus. Sugere-se uma avaliação mais aprofundada na interação entre as espécies estudadas nesse ecossistema ornamental, nos diversos estágios fenológicos. O estado fisiológico e a morfologia da planta hospedeira determinarão a sua resistência inerente, bem como as condições de crescimento da população de afídeos (BЕTHKE et al. 1998 apud SogLiA et al. 2003). A interação variedade resistente e controle biológico, precisa ser conhecida (LARA 1991) uma vez que a planta pode afetar diretamente os inimigos naturais ou indiretamente, afetando o herbívoro.

Após avaliadas as amostragens de $S$. articulatus e $P$. egena nos diversos quadrantes de $M$. communis durante os estádios vegetativo e de florescimento/frutificação, concluiu-se que a cochonilha pode ser utilizada como fonte de criação da joaninha. Por outro lado a planta, pela infestação da cochonilha, pode ser indicada como planta isca e pode, ou não, ser cultivada próxima às culturas de importância econômica, pois funciona como um reservatório da praga ou do inimigo natural.

\section{REFERENNCIAS}

Bartra, C. E. P. 1974. Biologia de Selenaspidus articulatus (Morgan) y sus principales controladores biologicos. Revista Peruana de Entomología 17: 60-68.

Batista-Filho, A.; L. G. Leite \& L. A. M. Machado. 1992. Manual de Controle Biológico. Rio de Janeiro, Sociedade Nacional de Agricultura, $56 \mathrm{p}$

Bergmann, E. C.; M. F. Stradioto \& A. D. Brisolla. 1998. Ocorrência de Selenaspidus articulatus (Morgan, 1889) em cultura de seringueira (Hevea brasiliensis Muell. Arg.) no município de Olimpia, Estado de São Paulo. Biológico 54: 1-6; 27-28.

CAmpos, M. S. 1993. Effect of biotic and abiotic factors on the settling percentage of Selenaspidus articulatus (Morg)(Coccoidea: Diaspididae). Revista de Protección Vegetal 2: 129-132.

Canales-Canales, A. \& L. Valdivieso-Jara. 1999. Handbook for biological control in olives. Jesus Maria, Servicio Nacional de Sanidad Agraria, $37 \mathrm{p}$.

Fontenla Rizo, J. L. 1990. Coexistence relationships of six species of Coccoidea (Insecta: Homoptera) in mixed citrus. Poeyana 391: 123.

Galli, J. C.; F. A. de Albuquerque; R. V. Gallao \& R. A. Pinto. 1995. Strategies for IPM programs to control scale, false rust mite and aphid on citrus and the effect on predators. Revista de Agricultura 70(3): 249-268.

Gliessman, S. R. 2001. Agroecologia: processos ecológicos em agricultura sustentável / Stephen Gliessman. 2. ed. Porto Alegre, Ed. Universidade/UFRGS, 653 p.

Gravena, S. 1996. Biologia da joaninha Pentilia egena, p. 83. In: S. Gravena (ed.). Manejo Ecológico de Pragas Agrícolas. Informativo, v. 2, n. 7.

Gravena, S. 1999. Joaninha Pentilia egena, p. 213. In: S. Gravena (ed.) Manejo Ecológico de Pragas Agrícolas. Informativo, v. 5, n. 18.

Hernandez, M. \& M. Ceballos. 1987. New host for biorregulators of coccids on citricus. San jose de Las Lajas, Habana, Cuba. Revista de Protección Vegetal 2: 178-182.

Hoz-Gonzalez, M. T. 1995. Oviposition in Selenaspidus articulatus (Morgan) (Homoptera:Diaspididae). Revista de Biologia 9: 123125 .

IBARRA-NunEZ, G. 1990. Artropods associated with coffe trees in a mixed plantation in Soconusco, Chiapas, México. I. Variety and abundance. Folia Entomologica Mexicana 79: 207-23.

Lara, F. M. 1991. Princípios de resistência de plantas a insetos. $2^{a}$ ed. São Paulo, Ícone, 336 p.

LARANJeiRA, F. F. 1997. Infestação de cochonilha pardinha em sansão do campo, Mimosa caesalpiniaefolia Bent., usado como cerca viva em pomares de laranja. Cordeirópolis (SP). Bragantia 56(2): 289290.

Levin, J. 1978. Estatística Aplicada a Ciências Humanas. São Paulo, HARBRA, Ed. Harper \& Row do Brasil Ltda, 310 p.

Loayza, R. M. \& J. R. P. PARRa. 2001. Cochonilha pardinha, Selenaspidus articulatus (Morgan) ( Hemiptera : Diaspididae), p. 53-58. In: E. Vilela; R. A. Zucchi \& F. CANToR (eds.) Histórico e impacto das pragas introduzidas no Brasil. Ribeirão Preto, Holos, 173 p.

Malavolta, E.; G. C. Vitti \& S. A. De Oliveira. 1997. Avaliação do estado nutricional das plantas: princípios e aplicações. 2.ed. Piracicaba, POTAFOS, $319 \mathrm{p}$.

Mendes, S. M.; V. H. P. Bueno; L. M. Carvalho \& L. C. P. Silveira. 2003. Efeito da densidade de ninfas de Aphis gossypii (Glover, 1877) (Hemiptera, Aphididae) no consumo alimentar e aspectos biológicos de Orius insidiosus (Say, 1832) (Hemiptera: Anthocoridae). Revista Brasileira de Entomologia 47(1): 19-24.

Oliveira, J. E. M.; J. B. Torres; A. F. Carrano-Moreira \& J. C. Zanuncio. 2001. Efeito da Densidade de Presas e do Acasalamento na Taxa de Predação de Fêmeas de Podisus nigrispinus (Dallas) (Heteroptera: Pentatomidae) em condições de Laboratório e Campo. Neotropical Entomology 30: 647-654.

Ометто, J. C. 1981. Bioclimatologia Vegetal. São Paulo. Ed. 
Agronômica Ceres, $425 \mathrm{p}$.

Perruso, J. C. \& P. C. R. Cassino. 1993. Flutuação Populacional de Selenaspidus articulatus (Morgan) (Hemiptera:Diaspididae) em Citrus sinensis (L.) no Estado do Rio de Janeiro. Anais da Sociedade Entomológica do Brasil 22: 401-404.

Perruso, J. C. \& P. C. R. Cassino. 1997. Plano de amostragem presençaausência para Selenaspidus articulatus (Morg.) (Homoptera.: Diaspididae) na cultura da laranjeira. Anais da Sociedade Entomológica do Brasil 26: 321-326.

Primavesi, A. M. 1990. Manejo Ecológico de Pragas e Doenças: Técnicas Alternativas para a Produção Agropecuária e Defesa do Meio Ambiente. $1^{\text {a }}$ ed. São Paulo, Nobel, 137 p.

Rodrigues, W. C. \& P. C. R. CAssino. 2004. Inimigos naturais, p. 97-114. In: P. C. R. Cassino \& W. C. Rodrigues (eds.). Citricultura Fluminense: principais pragas $e$ inimigos naturais. Seropédica, Ed. Universidade Rural (EDUR), 168 p.

Soglia, M. C. da M.; V. H. P. Bueno; S. M. M. Rodrigues \& M. V. Sampaio. 2003. Fecundidade e longevidade de Aphis gossypii (Glover, 1877) (Hemiptera, Aphididae) em diferentes temperaturas e cultivares comerciais de crisântemo (Dendranthema grandiflora Tzvelev. Revista Brasileira de Entomologia 47(1): 49-54.

SuRIs, M. 1999. Disposicion espacial de Selenaspidus articulatus Morg. (Coccoidea: Diaspididae) en naranjo Valencia (Citrus sinensis L.).
Revista de Protección Vegetal 14(1): 17-22.

Suris, M. \& I. Varona. 1998. Spatial distribution of Selenaspidus articulatus (Coccoidea:Diaspididae) in a Valencia orange grove. Revista de Protección Vegetal 3: 38-44.

Watanabe, M. A., C. Yoshil \& R. C. Siloto. 1994. Parasitismo em cochonilha Selenaspidus articulatus (Hemiptera: Diaspididae) (Morgan, 1889) em citrus nas regiões de Jaguariúna e Limeira-SP. Revista de Agricultura 69(2): 193-200.

Watanabe, M. A.; F. J. Tambasco; E. A. B. De Nardo; R. I. Viana \& G. D. Pereira. 2000a. Competition between Selenaspidus articulatus and Parlatoria ziziphi scales in orchards in the citrus region of São Paulo State. Laranja 21(1): 81-97.

Watanabe, M. A.; F. J. Tambasco; V. A. Costa; E. A. B. Nardo \& R. Facanali. 2000b. Population dynamics of some armored scale in citros trees in different São Paulo State localities, Brazil. Laranja 21(1): 49-64.

Williams, D. J. \& G. W. Watson. 1988. The scale insects of the tropical south Pacific Region. Part I- The armoureds scales (Diaspididae). London, International Institute of Entomology, $290 \mathrm{p}$.

Willink, M. C. R. DE \& L. E. Claps. 2003. Cocninillas (Hemiptera: Coccidae) presentes en plantas ornamentales de la Argentina. Neotropical Entomology 32(4): 625-637.

Recebido em 10.X.2003; aceito em 15.VII.2004 